\title{
Mueller matrix ellipsometry for enhanced optical form metrology of sub-lambda structures
}

Tim Käseberg, Johannes Dickmann, Thomas Siefke, Matthias Wurm, Stefanie Kroker, et al.

Tim Käseberg, Johannes Dickmann, Thomas Siefke, Matthias Wurm, Stefanie Kroker, Bernd Bodermann, "Mueller matrix ellipsometry for enhanced optical form metrology of sub-lambda structures," Proc. SPIE 11057, Modeling Aspects in Optical Metrology VII, 110570R (21 June 2019); doi: $10.1117 / 12.2527419$ 


\title{
Mueller matrix ellipsometry for enhanced optical form metrology of sub-lambda structures
}

\author{
Tim Käseberga ${ }^{\mathrm{a}}$, Johannes Dickmann ${ }^{\mathrm{a}}$, Thomas Siefke ${ }^{\mathrm{a}, \mathrm{b}}$, Matthias Wurmª, Stefanie Kroker ${ }^{\mathrm{a}, \mathrm{c}}$, \\ and Bernd Bodermann ${ }^{\mathrm{a}}$ \\ aPhysikalisch-Technische Bundesanstalt, Bundesallee 100, 38116 Braunschweig, Germany \\ ${ }^{\mathrm{b}}$ Friedrich-Schiller-Universität Jena, Abbe Center of Photonics, Institute of Applied Physics, \\ Max-Wien-Platz 1, 07743 Jena, Germany \\ 'Technische Universität Braunschweig, Laboratory for Emerging Nanometrology, \\ Mendelssohnstr. 3, 38106 Braunschweig, Germany
}

\begin{abstract}
Accurate metrology of nanostructures gains more and more importance and for efficiency reasons optical methods play a significant role here. Unfortunately, conventional optical microscopy is subject to the well-known resolution limit. The necessity to resolve objects smaller than this limit led to the development of superresolution methods which however are barely used in metrology for practical reasons. Non-imaging indirect optical methods like scatterometry and ellipsometry however are not limited by diffraction and are able to determine the critical dimensions of nanostructures. We investigate the application of different approaches for specifically manipulated near-fields in Mueller matrix ellipsometry to achieve an enhanced sensitivity for polarization based sub-wavelength topological information. To this end, we present first numerical simulations of these approaches. To examine the relationship between structural properties and Mueller matrix elements we designed individual structures based on geometrical shapes of varying parameters as well as small arrays. They are realized by lithography as holes in PMMA resist. First, we characterize SEM images of the structures to validate the fabrication process. Numerical simulations of the Mueller matrices of the structures by finite element method are discussed. Results indicate that conventional Mueller matrix ellipsometry alone is unsuitable but the extension to imaging Mueller matrix microscopy is promising for the characterization of sub-wavelength features.
\end{abstract}

Keywords: Ellipsometry, Mueller matrix, Surface plasmons, Plasmonic lenses

\section{INTRODUCTION}

Semiconductor industries are one of the most important economic sectors today. With the ongoing advances in the fabrication of nanostructures, transistors and chips get smaller and smaller, reaching just a few nanometers in length. ${ }^{1-3}$ But as fabrication techniques emerge, the characterization of nanostructures remains an important task more than ever. To ensure the correct fabrication of structures such small, highly precise measurement methods are required. While atomic force microscopy (AFM) is accurate but unsuited for the characterization of large areas, and scanning electron microscopy (SEM) is fast but expensive and potentially destructive, optical techniques enable fast, non-invasive measurements of large sample areas. ${ }^{4,5}$ Unfortunatly, conventional microscopy faces the resolution limit which is often associated with the Rayleigh criterion: ${ }^{6}$

$$
r=\frac{0.61 \lambda}{\mathrm{NA}}
$$

which defines the smallest distance $r$ between two objects that can just be observed seperately by light of the wavelength $\lambda$ in a system with the numerical aperture NA. For any optical system, it is impossible to resolve objects that are closer to each other than this smallest distance. However, modern technology readily enables the fabrication of structures that are way smaller than this limit. The demand to characterize these structures

Further author information: (Send correspondence to T.K.)

T.K.: E-mail: tim.kaeseberg@ptb.de

Modeling Aspects in Optical Metrology VII, edited by Bernd Bodermann, Karsten Frenner, Proc. of SPIE Vol. 11057, 110570R · C 2019 SPIE · CCC code: 0277-786X/19/\$21 · doi: 10.1117/12.2527419 


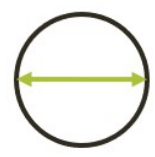

$500 \mathrm{~nm}$

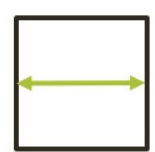

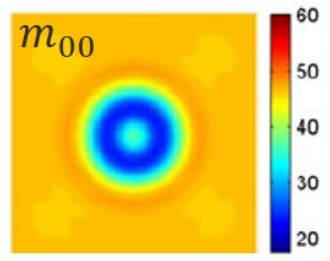

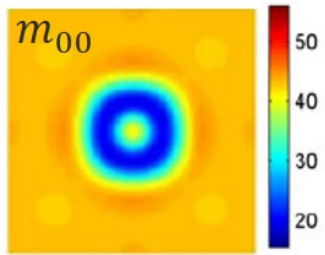

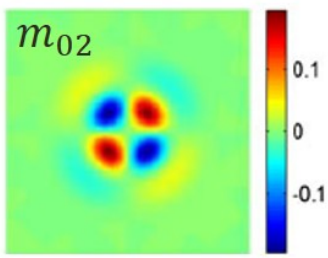

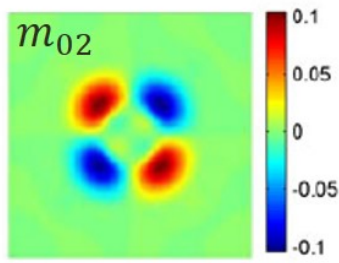

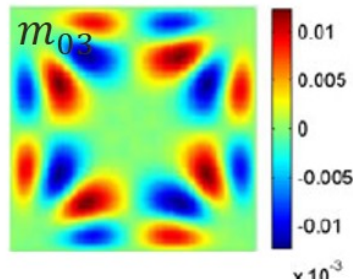

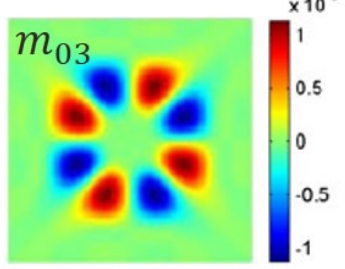

Figure 1. Numerically simulated images of selected Mueller matrix elements for a circle and a square structure of the same width, taken from Ref. 16.

lead to the development of superresolution microscopy methods. A quite recent as well as prominent example is the so-called stimulated emission depletion microscopy (STED) which was honored with the Nobel Prize in Chemistry 2014. ${ }^{7}$ The working principle of STED relies on the depletion of fluorescent regions with a torusshaped excitation spot while the area inside the torus is still active. This leads to a modification of the resolution limit and allows spatial resolution down to a few tens of nanometers in the far field. ${ }^{8}$ However, the intensity needed for the depletion can lead to photobleaching of the needed fluorophores. Moreover, the necessity of fluorophores in the first place makes this technique impractical in non-biological applications, particularly in metrology. In fact, many other super-resolution methods like photo-activated localization microscopy (PALM) or stochastic optical reconstruction microscopy (STORM) rely on the presence of fluorophores as well. ${ }^{9,10}$ In contrast to common microscopy methods, ellipsometry is an indirect optical measurement technique. It makes use of the otherwise obstructive diffraction and is already widely used to characterize dimensions down to a few Ångström. ${ }^{11,12}$ As it is not inherently limited by diffraction, ellipsometry is a promising candidate for new ways in terms of optical characterization. Especially Mueller matrix ellipsometry, which determines the Mueller matrix that describes the polarization properties of the sample, offers many opportunities for the metrology of nanostructures. ${ }^{13-15}$ Numerical simulations of Mueller matrix images of basic geometric structures have shown that off-diagonal matrix elements present enhanced sensitivity to sub-wavelength features compared to their overall intensity. ${ }^{16}$ An example for this is outlined in Figure 1, where two individual nanostructures, a circle and a square, cannot be distinguished well by their intensity image alone, but show clearly distinctive features in their off-diagonal Mueller matrix elements. Our goal is to exploit this behaviour in advanced ellipsometry setups for measurements beyond the classical resolution limit. In order to further enhance the sensitivity to sub-wavelength features, we want to combine the setup with approaches of shaped and manipulated near fields by placing specially designed nanostructures in the vicinity of the sample. Our current approach concentrates on the use of planar plamonic lenses as a near-field focusing tool.

In Section 2 we describe the working principle of basic planar plamonic lenses. Additionally, we present design examples for the application of plasmonic lenses in ellipsometry setups at visible wavelengths. The reference structures that we designed are presented in Section 3. Their choice and fabrication are discussed and evaluated by characterization of SEM images. Afterwards, in Section 4 numerical simulations of the reference structures are discussed.

\section{PLASMONIC LENS APPROACH}

The optical near-field contains a not negligible amount of high spatial frequency information about the topology of the sample under investigation. Unfortunately, the near-field decays exponentially with the distance from the sample and is hard to measure therefore. One technique that captures the otherwise lost information by scanning a small tip close to the sample is the near-field scanning optical microscopy (NSOM). However, scanning processes 
are often time consuming and the production of suitable tips can be cumbersome. Other approaches to enhance the resolution rely on so-called superlenses, which are metamaterial based lenses that reach resolutions beyond the classical limit. For some years, plasmonic lenses are promising objects of research and feature innovative developments to this day. ${ }^{17-21}$ These lenses are capable of focusing light with very small spot sizes, smaller than the incident wavelength. For this reason, we investigate ways to implement such a plasmonic lens into a Mueller matrix ellipsometry setup to gain higher resolution.

\subsection{Theoretical Framework}

A planar plasmonic lens typically consists of a slab of a material with negative permittivity like metal surrounded by a material with a positive permittivity like air and several slits through the slab like depicted in Figure $2 .{ }^{22,23}$ The thickness of the slab typically does not exceed the wavelength of the incident light and the width of the slits lies in the range of 30 to $200 \mathrm{~nm}$. When the slab is illuminated by transverse magnetic (TM) polarized light at the wavelength $\lambda_{0}$, surface plasmon polaritons (SPPs) may be excited which travel along the surface of the metal. ${ }^{17}$ While the slits are too narrow for light to transmit efficiently, SPPs may propagate through the slits as waveguide modes. Their propagation $\beta$ is proportional to the width $w$ of the corresponding slit: ${ }^{24}$

$$
\tanh \left(\frac{w}{2} \sqrt{\beta^{2}-k_{0}^{2} \varepsilon_{\mathrm{d}}}\right)=-\frac{\varepsilon_{\mathrm{d}} \sqrt{\beta^{2}-k_{0}^{2} \varepsilon_{\mathrm{m}}}}{\varepsilon_{\mathrm{m}} \sqrt{\beta^{2}-k_{0}^{2} \varepsilon_{\mathrm{d}}}},
$$

where $\varepsilon_{\mathrm{m}}$ and $\varepsilon_{\mathrm{d}}$ are the relative permittivity of the metal and the dielectric in the slits, respectively, and $k_{0}=\frac{2 \pi}{\lambda_{0}}$ is the vacuum wavenumber. After passing the slit, the SPPs transform back into propagating waves. Hence, a set of slits of different widths can be designed which generate a phase delay $\phi$ on the other side of the metallic slab, essentially depending only on the thickness $d$ of the slab: ${ }^{17}$

$$
\phi=\beta \cdot d .
$$

By choosing a set of slits of varying widths, like illustrated in Figure 2, it is possible to create a phase distribution that leads to a curved wave front and by this in a focal spot. To achieve this, the phase delay has to obey the following dependence on the position $x$ on the slab: ${ }^{17}$

$$
\phi(x)=2 n \pi+\frac{2 \pi f}{\lambda_{0}}-\frac{2 \pi \sqrt{f^{2}+x^{2}}}{\lambda_{0}},
$$

where $f$ is the desired distance between the lens and the focal spot, the focal length, and $n \in \mathbb{N}$. The propagation $\beta$ can be calculated using this equation. Thereby, the required slit width $w$ to achieve the corresponding phase delay in dependence of the position $x$ can be obtained.

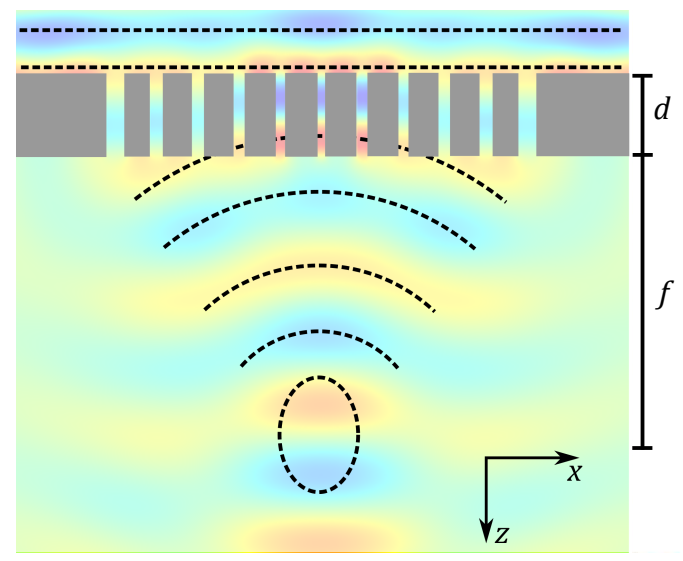

Figure 2. Basic structure of a planar plasmonic lens, built from a metallic slab (gray) with several slits. Light propagates faster through wider slits, resulting in a curved wavefront. 


\subsection{Lens Design}

We used the principle outlined in Section 2.1 to design plasmonic lenses for different wavelengths from the visible to the near-infrared range and for different focal lengths. To verify the optical functionality of the designs, we performed numerical simulations using JCMsuite, a finite element method (FEM) tool. ${ }^{25}$ Figure 3(a) and (b) show two examples for plasmonic lenses made of iridium for different wavelengths and a focal length of $5 \mu \mathrm{m}$. As SPPs are TM modes, the real part of the intensity of the magnetic field is depicted. The focal spots are clearly visible and their full widths at half maximum (FWHM) are smaller than a third of the incident wavelength. The focal length was chosen for practical reasons as larger focus lengths would be possible but accompanied with large computational costs. As a brief remark: As one-dimensional lines, these lenses would behave like perfect linear polarizers as only TM polarized light can exite SPPs. Additionally, they would focus like cylindrical lenses. However, when arranging the slits as rings of different widths, the polarisation of the incident light would be irrelevant and they would produce a focal spot like a spherical lens.

The classic planar plasmonic lens consists of a metallic slab with several small slits. As the production of such lenses can be challenging, especially for thicker slabs, we developed another lens design which is presented in Figure 4. Instead of a purely metallic slab, the lens now mostly consists of air. The slits were replaced by fins which are made of the substrate dielectric, preferably silica $\left(\mathrm{SiO}_{2}\right)$. The sidewalls of these fins are coated with a metal. The SPPs now do not propagate through slits of air but through the dielectric fins. Their propagation is ensured by the metallic coating which needs to be at least as thick as the penetration depth of the SPPs which corresponds to the skin depth at the metal-dielectric interface: ${ }^{26}$
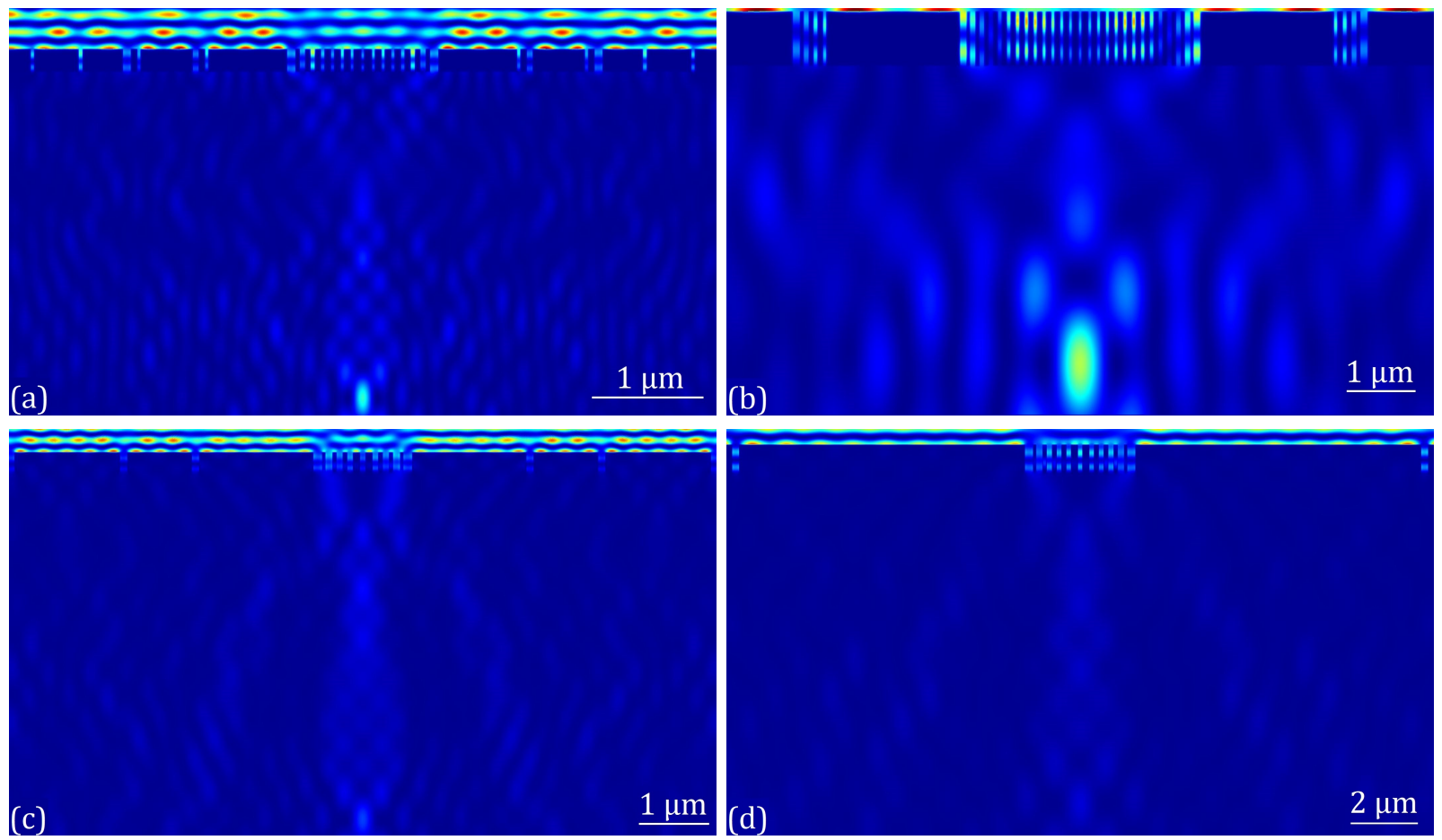

Figure 3. Examplary plasmonic lenses, cutout of intensity images. Lenses are located in the top of the images and light is send through them from the top. (a) Plasmonic lens for visible light $(\lambda=532 \mathrm{~nm})$, iridium slab thickness $d=370 \mathrm{~nm}$. The FWHM of the focal spot is not larger than $160 \mathrm{~nm}$. (b) Plasmonic lens for infrared light $(\lambda=1550 \mathrm{~nm})$, iridium slab thickness $d=950 \mathrm{~nm}$. The FWHM of the focal spot is not larger than $480 \mathrm{~nm}$. (c) Inverted plasmonic lens for visible light $(\lambda=532 \mathrm{~nm})$, focallength $f=5 \mu \mathrm{m}$ and fin height $d=300 \mathrm{~nm}$. The FWHM of the focal spot is not larger than $200 \mathrm{~nm}$. (d) Inverted plasmonic lens for infrared light $(\lambda=1550 \mathrm{~nm})$, focallength $f=10 \mu \mathrm{m}$ and fin height $d=900 \mathrm{~nm}$. The FWHM of the focal spot is not larger than $600 \mathrm{~nm}$. 


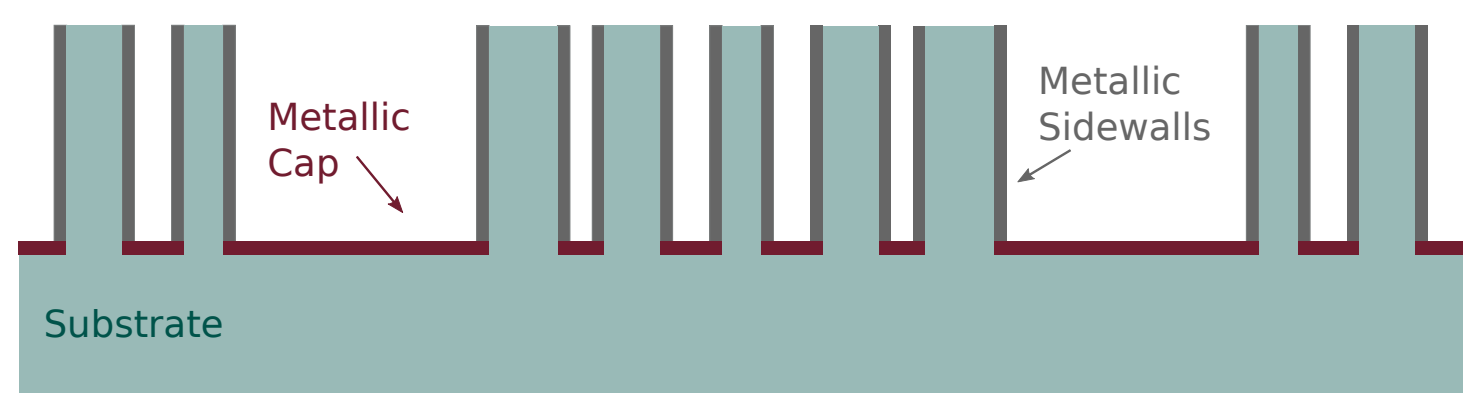

Figure 4. Inverted plasmonic lens design.

$$
z=\sqrt{\frac{\Re\left(\varepsilon_{\mathrm{m}}\right)+\varepsilon_{\mathrm{d}}}{\varepsilon_{\mathrm{m}}^{2}}} \frac{1}{k_{0}} .
$$

This way, interaction between adjacent fins is prevented as well. The substrate between the fins also needs to be capped with a layer of metal to prevent direct transmissions which would interfere with the SPPs. This design is easier to fabricate as fins in a dielectric are usually easier to etch than slits in several hundreds of nanometers of metal. The metallic coating can quite easily be realised by means of atomic layer deposition. Figure 3(c) and (d) present two examplary inverted plasmonic lens designs for the application at visible $(\lambda=532 \mathrm{~nm})$ and infrared $(\lambda=1550 \mathrm{~nm})$ wavelengths, similar to the classic designs in Figure 3(a) and (b). The substrate material for both lenses is $\mathrm{SiO}_{2}$ and for the metallic cap and sidewalls iridium was chosen for fabricational reasons. The coating is $35 \mathrm{~nm}$ thick and therefore larger than the penetration depth of the SPPs. The FWHM of the focal spot is still smaller than the wavelength of the incident light for both lenses. However, despite a similar design, the intensity of the focal spot decreased in comparison to the classic design. This is most likely due to the SPPs now propagating through $\mathrm{SiO}_{2}$ instead of air. Additionally, the design process for this kind of lens is still open for optimization.

\section{REFERENCE SAMPLE DESIGN}

The plasmonic lenses presented in Section 2 are planned to be used in an advanced Mueller matrix ellipsometry setup. This setup aims to obtain information about spatial features smaller than the wavelength of the incident light by evaluating the Mueller matrix. To get a deeper understanding of how certain elements of the Mueller matrix are connected to changes in the geometry of the structure, we intend to use particular references that are presented in this section.

\subsection{Structure Properties}

In order to understand the relationship between structural properties and the elements of the Mueller matrix, we designed a set of individual nanostructures, consisting of basic geometrical shapes of varying parameters. The shapes are depicted in Figure 5. In the style of the work by Kerwien ${ }^{16}$ illustrated in Figure 1 we chose basic square and circle shapes with different sizes (B and C) as well as squares presenting corner rounding of different radii (A) to emulate the transition from a square to a circle. The structures are meant to be individual which means that they are spaced by several millimeters to the next structure on the wafer later on to prevent any interactions. Apart from these individual structures, we are interested in the influence of small arrays with only a few structures (D). We fabricated these structures as holes in polymethylmethacrylate (PMMA) resist on a 100 $\mathrm{mm}$ silicon wafer using electron beam lithography. Following the work by Kerwien ${ }^{16}$ the holes are $100 \mathrm{~nm}$ deep. Silicon was chosen as a substrate material to enable ellipsometric measurements in reflection. With the holes being in the resist on top of the silicon substrate, we retain the option to later on etch the holes into the substrate to perform the same measurements with holes in silicon. A first test sample containing fields with different dose factors was processed to optimize lithography fabrication parameters. This sample is shown in Figure 6 . The influence of the dose factor on the size of the fabricated structures was examined and is discussed Section 3.2. Additionally, structural parameters are varied over the fields of the sample. The 'A' labeled rows contain square 
structures with a width $w$ of $5 \mu \mathrm{m}$ and varying corner radii $r_{\mathrm{c}}$. The square structures in the 'B' labeled rows as well as the circle structures in the ' $\mathrm{C}$ ' labeled rows vary in size $w$ or in radius $r$, respectively. The small arrays in the 'D' labeled rows are made up of $N^{2}$ square structures of width $w=1 \mu \mathrm{m}$ and with different pitches $\Lambda$ between the structures. Each field on the wafer is divided in nine equal sections for comparison as shown in Figure 6. The sections in the upper half of the wafer, apart from the ' $D$ ' labeled row, contain nine structures, each one processed with a different dose factor. The sections in the lower half of the wafer contain only one structure each. Between the sections as well as between the fields, cross marks for localizing the structures are placed. The specifications of the structure parameters are listed in Table 1.

A

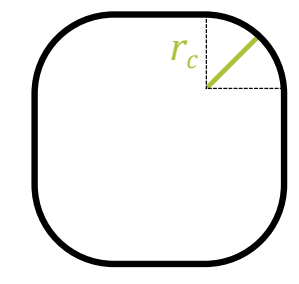

B

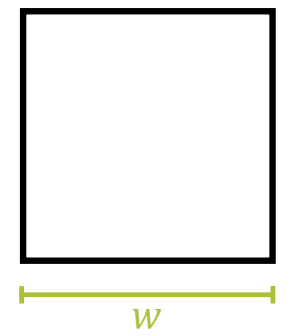

C

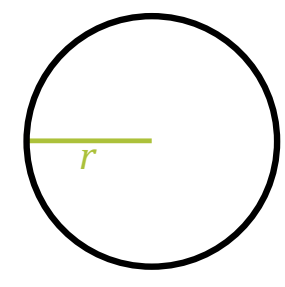

D

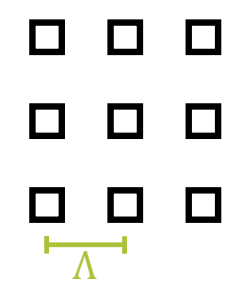

Figure 5. Basic geometrical shapes of the reference structures.
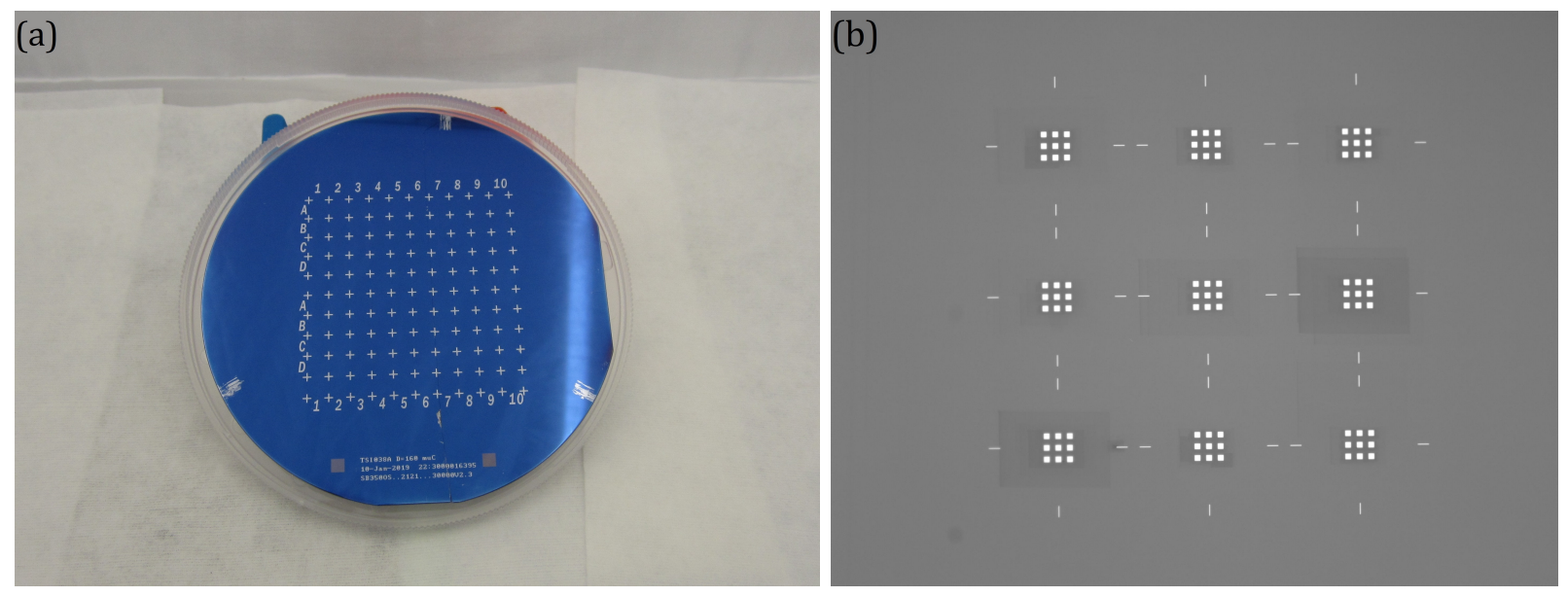

Figure 6. First test sample. The complete wafer (a) and a microscope image of field A1 on the upper half of the wafer (b). The sections are processed identically and contain nine structures each fabricated with different dose factors.

Table 1. Structure specifications of the designed reference structures.

\begin{tabular}{|c|l|c|c|c|c|c|c|c|c|c|c|}
\hline & & 1 & 2 & 3 & 4 & 5 & 6 & 7 & 8 & 9 & 10 \\
\hline $\mathrm{A}$ & $r_{\mathrm{c}} / \mathrm{nm}$ & 100 & 150 & 200 & 300 & 400 & 500 & 750 & 1000 & 1500 & 2000 \\
\hline $\mathrm{B}$ & $w / \mathrm{nm}$ & 50 & 70 & 100 & 200 & 250 & 500 & 750 & 1000 & 1500 & 2000 \\
\hline $\mathrm{C}$ & $r / \mathrm{nm}$ & 25 & 35 & 50 & 100 & 125 & 250 & 375 & 500 & 750 & 1000 \\
\hline $\mathrm{D}$ & $N, \Lambda / \mu \mathrm{m}$ & $1,-$ & 2,10 & 3,10 & 4,10 & - & $1,-$ & 2,20 & 3,20 & 4,20 & - \\
\hline
\end{tabular}




\subsection{SEM Sample Characterization}

In the electron beam lithography fabrication process, structures were produced with varying dose factors to determine which factor leads to an ideal shape concerning structure size as well as corner radius. Scanning electron microscope (SEM) images of the structures were made to examine the influence of the dose factor on their shape. Figures 7 and 8 present some examples of these SEM images. As more than 5000 SEM images were made in the process, the characterization of these images was automated using a custom written Python 3.6 script. The script uses the Canny edge detection algorithm and the Hough transform implementation of the scikit-image package ${ }^{27-29}$ to detect edges in the SEM image which were used to determine the dimensions of the structures. In the beginning, the Canny edge detection algorithm was used to obtain a binary image containing only edge points. For the square like structures (A, B, and D), their size was determined by performing a Hough line transform on this image to detect straight lines. An example is shown in Figure 9(b). Afterwards, the mean distances between vertical and horizontal lines were calculated, respectively. To detect edge points that belong to rounded corners like the one in Figure 9(a), the deviation from the second derivation of the edge points lateral position along the straight edges was evaluated until it was larger than a certain threshold value. The corner points chosen this way as well as the circle structures $(\mathrm{C})$ were then fitted to a circle. An example for such a least squares fit circle is shown in Figure 9(c).

Apart from the methods presented here, another evaluation method by Frase et al. which is based on a physical model of the edges and their appearance in SEM images was considered. ${ }^{30}$ However, the differences in the many images processed with varying dose factors were large enough so that the great accuracy of this method was set aside in favour of a higher speed of evaluation. Apart from that, the algorithm of Frase et al. only deals with the measurement of line widths but does not allow the evaluation of circles or curves.

Concerning the dose factors, small factors lower than 1.5 proved to be best suited for the fabrication of the structures. For the small squares (B) and circles (C) with sizes below $100 \mathrm{~nm}$, the electron beam lithography process came to its limits and was not able to correctly realise the desired structures. Especially the square structures turned out to rather be circles in this size range. Therefore, these structures will be produced by another lithography technique later on. Anyway, apart from their appearance, their overall size could be reached best with dose factors between 1.5 and 2.0 in this size range. For much larger structures, a factor of 1.0 was usually sufficient. However, the process often seems to exceed the desired corner radii of the rounded squares (A). Especially for smaller radii, the correct fabrication of rounded corners seems challenging. Thermal scanning probe lithography, which is going to be used for the smaller structures later on, would probably be suited for the fabrication of the rounded corners as well.
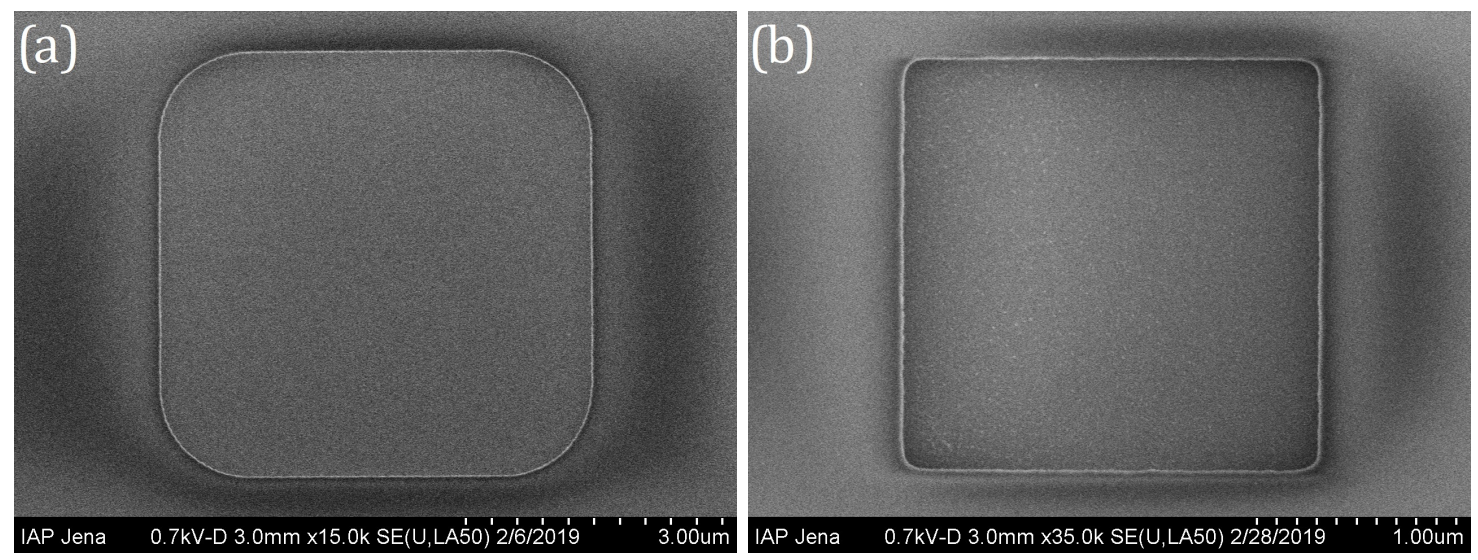

Figure 7. Examples for SEM images of a square presenting corner rounding (a) and a square (b) structure of the fabricated test sample. 

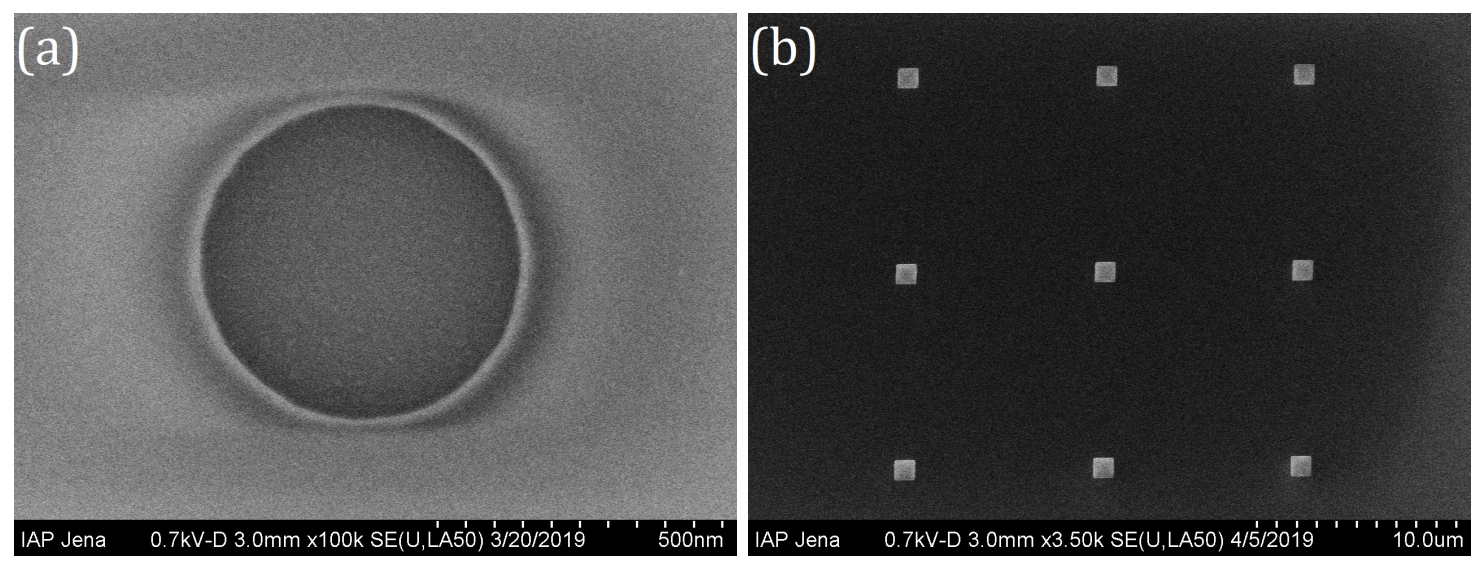

Figure 8. Examples for SEM images of a circle (a) and an array (b) structure.
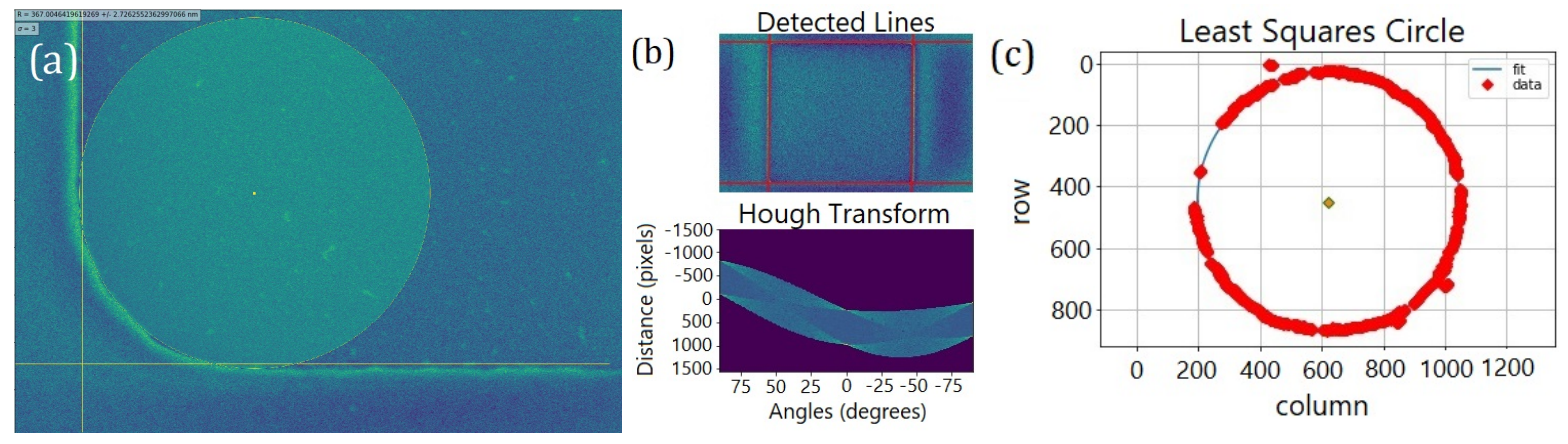

Figure 9. Examples for evaluated SEM images. (a) Rounded corner and the fit circle. Yellow lines indicate the points were the detected corner starts. Each point between the lines was used to fit the circle. (b) Detected straight edges (upper) and Hough space (lower) for a square structure. (c) Least squares fit of all edge points for a circle structure.

\section{NUMERICAL SIMULATIONS}

In order to correctly interpret ellipsometric measurements on the reference structures later on, it is necessary to perform numerical simulations which can be used to conclude on the shape of the sample. Like with the plasmonic lenses, we perform these simulations using JCMsuite. As an example, the mesh created for the numerical simulations of an individual square structure with corner rounding is depicted in Figure 10. The two-fold mirror symmetry of the structure was exploited to save computational costs. The Mueller matrix of this $5 \mu \mathrm{m}$ sized structure was simulated for different radii of the corner rounding using a plane wave at $\lambda=532$ $\mathrm{nm}$ wavelength and $\theta=30^{\circ}$ angle of incidence. A corner radius of $0 \mathrm{~nm}$ represents a perfect square with no corner rounding whereas a radius of $2500 \mathrm{~nm}$ means that the square degenerates into a circle. Figure 11 shows a first test of a simulated Mueller matrix depending on the corner radius of this structure in a transition from a square to a circle. As the whole structure was illuminated and the overall reflection was detected, the figure shows an accumulated result for the whole simulated area. The matrix elements are normalized to the first matrix element $m_{00}$. As expected, the elements on the main diagonal lie close to 1 and -1 whereas off-diagonal matrix elements tend to lie around 0 . This indicates no significant influence of the structure to the polarization of the incident light. Small influences of the corner rounding can be interpreted into the curve progression of some matrix elements for small radii, for example $m_{12}$ or $m_{21}$. In these elements, with increasing corner radius an increasing tendency can be observed. The trend decreases at a radius of about $1 \mu \mathrm{m}$ and later increases again at about $2 \mu \mathrm{m}$. Similar tendencies can be observed in other elements like $m_{02}$ or $m_{23}$, too. However, these progressions are governed by numerical inaccuracies due to a not yet fully optimized simulation process. These inaccuracies matter for example in $m_{30}$, where it is hard to decide whether the trend stems from genuine form effects or is rather induced by noise. These insecurities can be sorted out by simulations at higher precisions and 


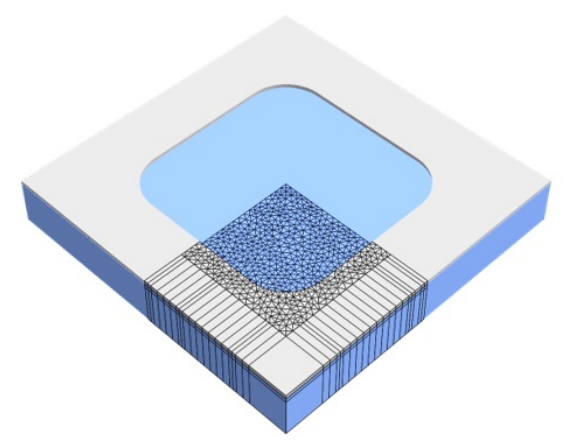

Figure 10. Mesh for a corner rounding structure, generated by the JCMwave software.

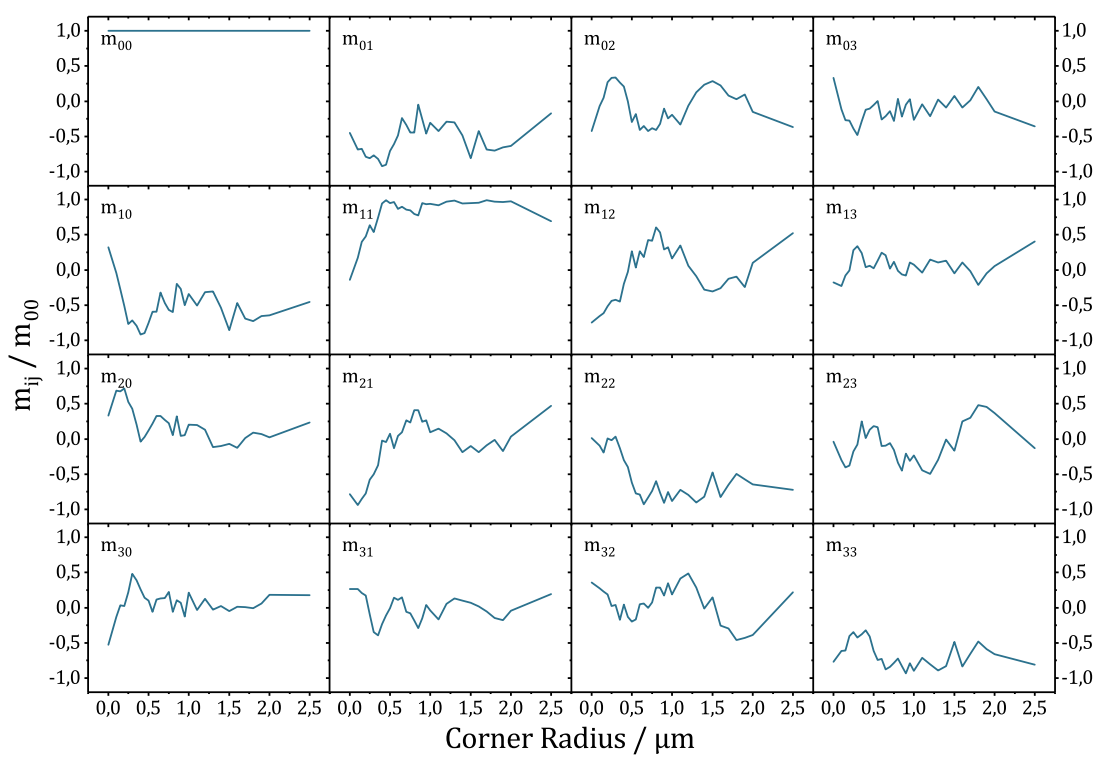

Figure 11. Preliminary Mueller matrix simulation for a square structure depending on corner radius at wavelength $\lambda=532 \mathrm{~nm}$ and $30^{\circ}$ incidence. The detection was assumed to be in reflection on the opposite side of the sample in a $30^{\circ}$-cone around the angle of reflection.

thereby higher computational costs. Additionally, simulations at other azimuthal angles, for example along the diagonal of the sample, would provide more information about the form influence on the matrix elements. Apart from that, it is still not possible to directly connect the Mueller matrix elements to the influence of the corner rounding alone as in this configuration, the Mueller matrix represents the whole structure and was obtained for a rather large acceptance angle. It is important to measure the scattered field in an angular range as broad as possible to obtain high spatial frequency information, but typical experimental setups will allow only small acceptance angles. These setups might be able to scan the half space above the sample in order to obtain more information, but this method is rather slow and would require additional post-processing. The results illustrate that an imaging ellipsometry setup to measure the Mueller matrix for each point in a microscope image in the style of Figure 1 is necessary to correctly connect features like the corner radius to progressions in the measured matrix elements. A microscopy imaging setup with a high numerical aperture would allow the measurement of the Mueller matrix spatially resolved at a high acceptance angle in a fast way. Additionally, support structures like the plasmonic lenses presented in Section 2 that further enhance the sensitivity of the ellipsometer to high spatial frequencies would be beneficial to implement. 


\section{CONCLUSION}

In this contribution, we presented a new design concept for plasmonic lenses complying fabricational difficulties and discussed their application in future advanced Mueller matrix ellipsometry setups. The design relies on metal coated dielectric fins for the propagation of SPPs. To this end, the focal spots that can be achieved are of lower intensity than for their classic counterparts, but their FWHM is still smaller than the incident wavelength and the final design will be further optimized. Furthermore, a set of reference structures has been design, fabricated and evaluated. As concluded from the SEM images, depending on the structure size, a dose factor of 1.0 to 1.5 would be sufficient for the correct fabrication of the structures. On the basis of these investigations, a final reference sample will be fabricated that is going to be used for reference measurements in the advanced ellipsometry setup. First numerical simulations of the reference structures show that a single Mueller matrix measurement of the whole structure alone is insufficient for the classification of geometrical features. Instead, a microscopical imaging setup would be needed in combination with support structures like the presented plasmonic lens to make effects in off-diagonal Mueller matrix elements more prominent.

\section{ACKNOWLEDGMENTS}

This work is funded through the project 17FUN01 "BeCOMe" within the Programme EMPIR. The EMPIR initiative is co-founded by the European Unions Horizon 2020 research and innovation program and the EMPIR Participating Countries.

\section{REFERENCES}

[1] Lee, H., Yu, L., Ryu, S., Han, J., Jeon, K., Jang, D., Kim, K., Lee, J., Kim, J., Jeon, S., Lee, G., Oh, J., Park, Y., Bae, W., Lee, H., Yang, J., Yoo, J., Kim, S., and Choi, Y., "Sub-5nm All-Around Gate FinFET for Ultimate Scaling," in [2006 Symposium on VLSI Technology, 2006. Digest of Technical Papers.], 58-59, IEEE, Honolulu (2006).

[2] Anthony, S., "IBM unveils world's first 5nm chip." Ars Technica, 05 June 2017 https://arstechnica.com/ gadgets/2017/06/ibm-5nm-chip/ (2017). (Accessed: 15 May 2019).

[3] Lu, W., Lee, Y., Murdzek, J., Gertsch, J., Vardi, A., Kong, L., George, S. M., and del Alamo, J. A., "First Transistor Demonstration of Thermal Atomic Layer Etching: InGaAs FinFETs with sub-5 nm Fin-width Featuring in situ ALE-ALD," in [2018 IEEE International Electron Devices Meeting (IEDM)], 39.1.1-39.1.4, IEEE, San Francisco (2018).

[4] Binnig, G., Quate, C. F., and Gerber, C., "Atomic force microscope," Phys. Rev. Lett. 56, 930-933 (1986).

[5] Smith, K. C. A. and Oatley, C. W., "The scanning electron microscope and its fields of application," Br. J. Appl. Phys. 6(11), 391-399 (1955).

[6] Brooker, G., [Modern Classical Optics], Oxford University Press, Oxford, corrected ed. (2014).

[7] "The Nobel Prize in Chemistry 2014." NobelPrize.org. Nobel Media AB 2019. https://www.nobelprize. org/prizes/chemistry/2014/press-release/ (2014). (Accessed: 15 May 2019).

[8] Hell, S. W. and Wichmann, J., "Breaking the diffraction resolution limit by stimulated emission: stimulatedemission-depletion fluorescence microscopy," Opt. Lett. 19(11), 780-782 (1994).

[9] Betzig, E., Patterson, G. H., Sougrat, R., Lindwasser, O. W., Olenych, S., Bonifacino, J. S., Davidson, M. W., Lippincott-Schwartz, J., and Hess, H. F., "Imaging intracellular fluorescent proteins at nanometer resolution," Science 313(5793), 1642-1645 (2006).

[10] Rust, M. J., Bates, M., and Zhuang, X., "Sub-diffraction-limit imaging by stochastic optical reconstruction microscopy (STORM)," Nature Methods 3, 793-796 (2006).

[11] Losurdo, M., "Applications of ellipsometry in nanoscale science: Needs, status, achievements and future challenges," Thin Solid Films 519(9), 2575-2583 (2011).

[12] Rothen, A., "The Ellipsometer, an Apparatus to Measure Thicknesses of Thin Surface Films," Rev. Sci. Instrum. 16(2), 26-30 (1945).

[13] Liu, S., Du, W., Chen, X., Jiang, H., and Zhang, C., "Mueller matrix imaging ellipsometry for nanostructure metrology," Opt. Express 23(13), 17316-17329 (2015). 
[14] Liu, S., Chen, X., and Zhang, C., "Development of a broadband mueller matrix ellipsometer as a powerful tool for nanostructure metrology," Thin Solid Films 584, 176-185 (2015).

[15] Tan, Y., Chen, C., Chen, X., Du, W., Gu, H., and Liu, S., "Development of a tomographic mueller-matrix scatterometer for nanostructure metrology," Rev. Sci. Instrum. 89(7), 073702 (2018).

[16] Kerwien, N., "On the influence of polarization effects in microscopic image formation," Berichte aus dem Insitut für Technische Optik, Stuttgart 58 (2007).

[17] Shi, H., Wang, C., Du, C., Luo, X., Dong, X., and Gao, H., "Beam manipulating by metallic nano-slits with variant widths," Opt. Express 13(18), 6815-6820 (2005).

[18] Xu, T., Du, C., Wang, C., and Luo, X., "Subwavelength imaging by metallic slab lens with nanoslits," Appl. Phys. Lett. 91(20), 201501 (2007).

[19] Chen, Q., "Effect of the number of zones in a one-dimensional plasmonic zone plate lens: Simulation and experiment," Plasmonics 6(1), 75-82 (2011).

[20] Li, H., Fu, L., Frenner, K., and Osten, W., "Cascaded plasmonic superlens for far-field imaging with magnification at visible wavelength," Opt. Express 26(8), 10888-10897 (2018).

[21] Li, H., Fu, L., Frenner, K., and Osten, W., "Cascaded DBR plasmonic cavity lens for far-field subwavelength imaging at a visible wavelength," Opt. Express 26(15), 19574-19582 (2018).

[22] Liu, Z., Steele, J. M., Srituravanich, W., Pikus, Y., Sun, C., and Zhang, X., "Focusing surface plasmons with a plasmonic lens," Nano Letters 5(9), 1726-1729 (2005).

[23] Verslegers, L., Catrysse, P. B., Yu, Z., White, J. S., Barnard, E. S., Brongersma, M. L., and Fan, S., "Planar lenses based on nanoscale slit arrays in a metallic film," Nano Letters 9(1), 235-238 (2009).

[24] Gordon, R. and Brolo, A. G., "Increased cut-off wavelength for a subwavelength hole in a real metal," Opt. Express 13(6), 1933-1938 (2005).

[25] "JCMsuite. The Simulation Suite for Nano-Optics." JCMwave GmbH, Berlin. https://www. jcmwave.com/ (2019). (Accessed: 15 May 2019).

[26] Barnes, W. L., "Surface plasmon-polariton length scales: a route to sub-wavelength optics," J. Opt. A 8(4), 87-93 (2006).

[27] Canny, J., "A computational approach to edge detection," IEEE Transactions on Pattern Analysis and Machine Intelligence PAMI-8(6), 679-698 (1986).

[28] Hough, P. V. C., "Machine Analysis of Bubble Chamber Pictures," Conf. Proc. C590914, 554-558 (1959).

[29] van der Walt, S., Schönberger, J. L., Nunez-Iglesias, J., Boulogne, F., Warner, J. D., Yager, N., Gouillart, E., Yu, T., and the scikit-image contributors, "scikit-image: image processing in Python," PeerJ 2, e453 (2014).

[30] Frase, C. G., Buhr, E., and Dirscherl, K., "CD characterization of nanostructures in SEM metrology," Measurement Science and Technology 18(2), 510-519 (2007). 